This item was submitted to Loughborough's Research Repository by the author.

Items in Figshare are protected by copyright, with all rights reserved, unless otherwise indicated.

\title{
Mesomechanical modelling of SnAgCu solder joints in flip chip
}

PLEASE CITE THE PUBLISHED VERSION

PUBLISHER

(C) Elsevier

LICENCE

CC BY-NC-ND 4.0

\section{REPOSITORY RECORD}

Gong, Jicheng, Changqing Liu, Paul P. Conway, and Vadim V. Silberschmidt. 2019. "Mesomechanical Modelling of Snagcu Solder Joints in Flip Chip”. figshare. https://hdl.handle.net/2134/3751. 
This item was submitted to Loughborough's Institutional Repository (https://dspace.lboro.ac.uk/) by the author and is made available under the following Creative Commons Licence conditions.

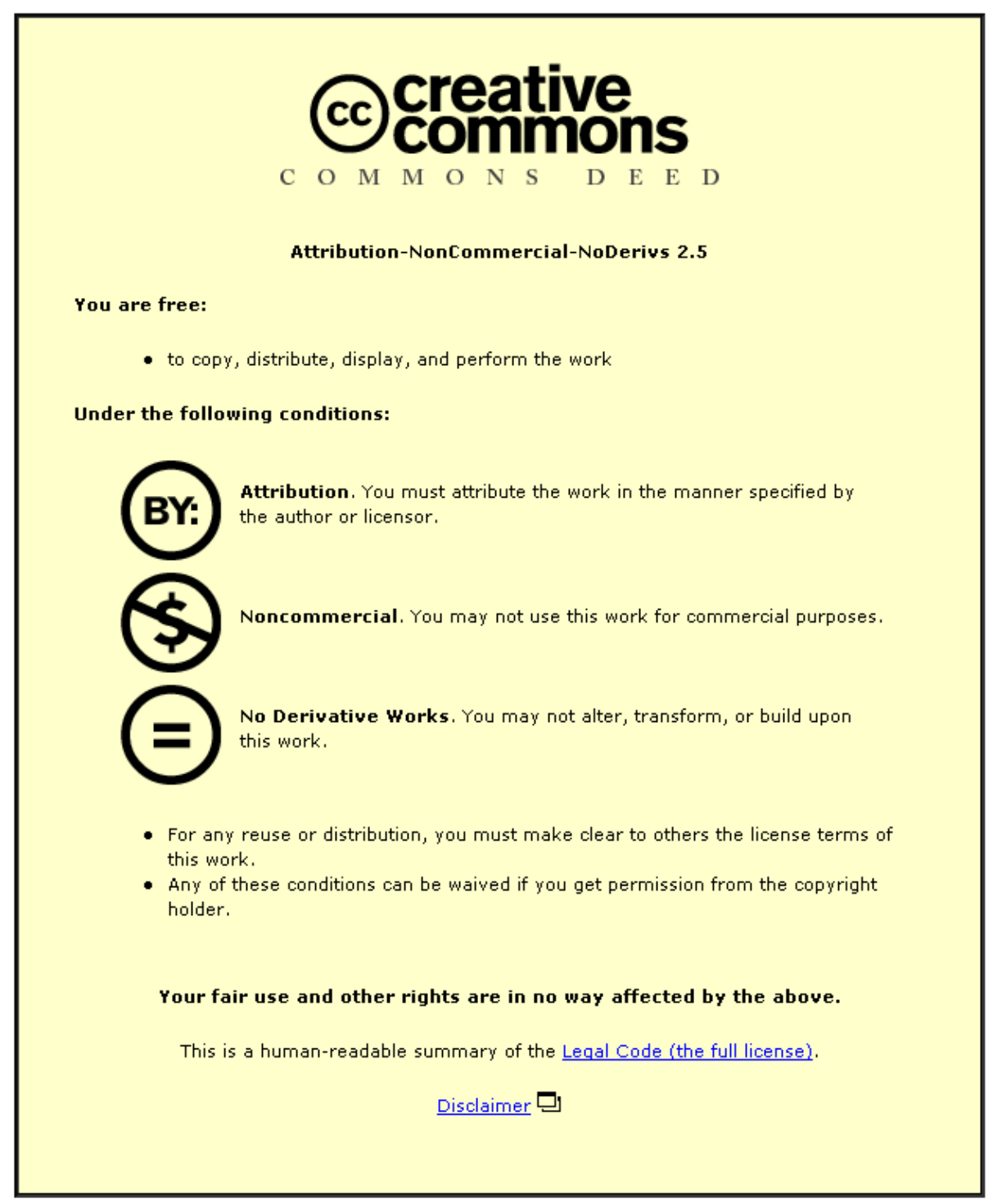

For the full text of this licence, please go to: http://creativecommons.org/licenses/by-nc-nd/2.5/ 


\title{
Mesomechanical Modelling of SnAgCu Solder Joints in Flip Chip
}

\author{
Jicheng Gong, Changqing Liu, Paul P. Conway, Vadim V. Silberschmidt \\ Wolfson School of Mechanical and Manufacturing Engineering \\ Loughborough University, Loughborough, LE11 3TU, UK
}

\begin{abstract}
In modern microelectronic packages (considered here as a mesoscale), the size of microstructural features of an alloy is compatible with the scale of an entire element that can contain only one or a few grains. In this case, the mechanical behaviour of the element deviates from isotropic/homogenous character at the macroscopic scale of a bulk specimen, comprising a large number of randomly oriented grains. Generally, a crystal-plasticity model, which is based on dislocation sliding in certain slip systems, is applied to describe a local lattice-induced anisotropic behaviour. However, even at a room temperature, the movement of dislocations is not a single mechanism of the inelastic behaviour of eutectic SnAgCu solder due to its low melting point. Under a low-magnitude loading condition, creep also has an effect due to a movement of vacancies. At high temperatures, this creep can become a dominant mechanism for the inelastic behaviour, diminishing the role of the crystal-plasticity model. This paper accounts for the creep component of deformation and unites it with the traditional crystal-plasticity model. In addition, deformation due to thermal expansion is introduced into the constitutive equation to capture the major mechanisms of the mechanical behaviour of a SnAgCu solder micro-joint used in electronics.
\end{abstract}

Key word: SnAgCu Solder, Crystal mechanics, Flip chip, Finite elements

\section{Introduction}

With the continuous increase in complexity of integrated circuits in electronic products, the 
size of solder joints for interconnections between components and a substrate has to decrease significantly to minimise the required area on the board. For instance, solder joints in a flip chip have been miniaturized to a length scale below $100 \mu \mathrm{m}$. The formation behaviour of SnAgCu grain is described in [1]. At such a small scale, a lead-free SnAgCu solder joint contains only one or a few grains, and therefore, its mechanical behaviour shifts from polycrystalline- to single crystal-based one [2, 3]. Generally, a crystal-plasticity model, which is based on dislocation sliding in certain slip systems of an alloy, is used to describe the plastic behaviour of a crystal [4]. However, the melting point of most solder materials for electronics is relatively low. For instance, the room temperature $\left(298^{\circ} \mathrm{K}\right)$ is 0.6 time of the melting point of SnAgCu eutectic alloy $\left(490^{\circ} \mathrm{K}\right)$. In this case, the major inelastic deformation mechanism of a solder crystal is creep rather than plasticity.

For most metals and alloys, there are two basic mechanisms that contribute to creep [5]: dislocation creep (due to movement of dislocations, e.g. dislocation glide and climb) and diffusional creep (due to vacancy diffusion, e.g. Nabarro-Herring creep and Coble creep). Each of these mechanisms is characterized by its stress exponent and activation energy in the power-law constitutive equation. For SnAgCu solder, it has different magnitudes of stress exponents at different stress regions [6]. Figure 1 gives a typical relationship between a creep strain rate and stress for $\mathrm{SnAgCu}$ solder at different temperatures. At high stress levels, where the creep rate has a high stress exponent in terms of the power law, creep due to a movement of dislocations is considered as the dominant mechanism and therefore can be described by a modified crystal-plasticity model [4]. However, in the low-stress region with a low stress exponent, the dominant creep mechanism is vacancy diffusion, the movement of which is directed by stresses rather than a sliding in slip systems. Both mechanisms contribute to the mechanical behaviour of a joint since variations of temperature and a subsequent change of loading conditions are inevitable in electronics due to the mismatch of coefficients of thermal 
expansion (CTEs) of materials forming a package. Therefore, the traditional crystal-plasticity model is not adequate for the mechanical behaviour of a SnAgCu micro joint with a few grains.

Besides elastic and inelastic deformations, another fundamental behaviour of solder materials in electronics is thermal expansion due to temperature variations in a package during its service or a thermal cycling test. For a micro joint, it is an essential source of the anisotropic behaviour for SnAgCu crystal, which is directly related to reliability of the joint [3], and cannot be neglected. This paper suggests a constitutive equation, which can account for all the major deformational mechanisms of a $\mathrm{SnAgCu}$ crystal, including the elastic behaviour, dislocation creep, diffusional creep and thermal expansion, so that it has the potential to predict the response of SnAgCu solder micro joints with few grains. Due to the lack of respective thermomechanical parameters for $\mathrm{SnAgCu}$ crystal, only the inelastic part is implemented in the model for discussion. The proposed model is integrated and implemented using the commercial finite element software ABAQUS with the subroutine UMAT. It is tested by application to a micro-joint of a flip chip package under a thermal cyclic load.

\section{Crystal model}

\subsection{Constitutive equation}

In electronics, mechanical loading on solder joints mainly results from the mismatch of CTEs of components they interconnect. For instance, in the flip chip electronic package, the CTEs of a silicon chip and PCB substrate are $2.8 \times 10^{-6} \mathrm{~K}^{-1}$ and $17 \times 10^{-6} \mathrm{~K}^{-1}$, respectively. A solder joint between them undergoes a shear loading when the temperature changes in the package. Within the range of the temperature excursions of the package in service, the thermal deformation is considerably small. Therefore, the constitutive equation is developed 
within the assumption of small deformations.

As analyzed above, there are three main sources of deformation for a SnAgCu crystal: elastic, creep and thermal expansion. Accordingly, the total strain $\varepsilon_{\text {total }}$ can be defined as a sum of elastic strain $\varepsilon_{\mathrm{e}}$, creep stain $\boldsymbol{\varepsilon}_{\mathrm{c}}$ and strain due to thermal expansion $\boldsymbol{\varepsilon}_{\mathrm{th}}$ :

$$
\boldsymbol{\varepsilon}_{\text {total }}=\boldsymbol{\varepsilon}_{\mathrm{e}}+\boldsymbol{\varepsilon}_{\mathrm{c}}+\boldsymbol{\varepsilon}_{\mathrm{th}} \text {. }
$$

For a bulk specimen, different creep behaviours of SnAgCu solder with a varying power law are generally unified by a hyperbolic sine equation [6], or described by a double power law [7]. In this paper, the model employs the concept of the double power law so that each type of creep behaviour can be treated individually. Accordingly, the creep strain is subdivided into Vacancy-Diffusion (VD) component $\boldsymbol{\varepsilon}_{\mathrm{vd}}$ and Dislocation-Movement (DM) component $\boldsymbol{\varepsilon}_{\mathrm{dm}}$ :

$$
\boldsymbol{\varepsilon}_{\mathrm{c}}=\boldsymbol{\varepsilon}_{\mathrm{vd}}+\boldsymbol{\varepsilon}_{\mathrm{dm}} .
$$

Following Hooke's law, stresses are expressed as

$$
\boldsymbol{\sigma}=\mathbf{C}: \boldsymbol{\varepsilon}_{\mathrm{e}},
$$

where $\mathbf{C}$ denotes the fourth-order elastic stiffness tensor. Here, $\mathbf{C}$ has 21 independent components, and can fully describe the anisotropic elastic behaviour of $\beta$-Sn matrix. In DM creep, deformations mainly result from dislocation glide, glide-climb-glide and pure climb mechanisms. The first two mechanisms can lead to a shearing deformation along certain slip systems. Note that a climbing process in glide-climb-glide creep, which is mainly due to the existence of intermetallic particles in $\beta$-Sn matrix, does not follow this shearing deformation. But it can lead to small deviations since climb is not the dominant process compared with glide due to the small volume fraction of intermetallic particles in eutectic SnAgCu solder. The last mechanism, pure climb, will be discussed together with VD creep. Therefore, the evolution of DM creep strains due to the first two mechanisms can be defined as the sum of 
the shear creep rates from slip systems:

$$
\dot{\boldsymbol{\varepsilon}}_{\mathrm{dm}}=\sum_{\alpha=1}^{N} \dot{\gamma}^{(\alpha)} \mathbf{s}^{(\alpha)},
$$

where $N$ is the number of slip system, $\dot{\gamma}^{(\alpha)}$ is the scalar shear strain rate of the $\alpha$ th slip system and $\mathbf{s}^{(\alpha)}$ is the Schmid tensor. Here, $\mathbf{s}^{(\alpha)}$ is expressed as:

$$
\mathbf{s}^{(\alpha)}=\frac{1}{2}\left(\mathbf{m}^{(\alpha)} \otimes \mathbf{n}^{(\alpha)}+\mathbf{n}^{(\alpha)} \otimes \mathbf{m}^{(\alpha)}\right),
$$

where $\mathbf{n}^{(\alpha)}$ is the slip direction and $\mathbf{m}^{(\alpha)}$ is the normal to the slip plane with $\left|\mathbf{m}^{(\alpha)}\right|=\left|\mathbf{n}^{(\alpha)}\right|=1$. The shear strain rate is a function of the resolved stress on the slip system, temperature and hardening variables. Taking the concept of steady-state creep, the effect of hardening is not considered, and the shear strain is expressed in the form of a power law:

$$
\dot{\gamma}^{(\alpha)}=A^{(\alpha)}\left(\tau^{(\alpha)}\right)^{n_{1}} \exp \left(-\frac{Q^{(\alpha)}}{R T}\right),
$$

where $A^{(\alpha)}$ is a constant, $n_{1}$ is the stress exponent, $Q^{(\alpha)}$ is the activation energy, $T$ is the absolute temperature, $R$ is the gas constant and $\tau^{(\alpha)}$ is the resolved shear stress on the slip plane in the slip direction:

$$
\tau^{(\alpha)}=\mathbf{s}^{(\alpha)}: \boldsymbol{\sigma}
$$

Diffusional creep is generally divided into two categories in terms of VD paths: along grain boundaries (Coble creep) and inside a grain (Nabarro-Herring creep). In a solder micro joint with few grains, there is either no grain boundary (in a single-crystal joint) or a few grain boundaries (e.g. in a bi-crystal joint). In this case, the Coble creep is expected to have a little effect, and therefore is not considered in this constitutive equation. In Nabarro-Herring creep, the movement of vacancies is controlled by the direction of stresses. Supposing that it is 
isotropic, the rate of its strain components is proportional to deviatoric stresses. The creep rate in the power law form is expressed as

$$
\dot{\varepsilon}_{\mathrm{vd}}^{i j}=\frac{3 S^{i j}}{2 \sigma_{\text {eq }}} A\left(\sigma_{\text {eq }}\right)^{n_{2}} \exp \left(-\frac{Q}{R T}\right),
$$

where $\mathrm{S}_{i j}$ is a deviatoric stress component, $n_{2}$ is the stress exponent and $\sigma_{\mathrm{eq}}$ is the equivalent stress. It should be mentioned that in DM, creep due to pure dislocation climb is also directed by stresses, and therefore can be introduced in the same way as that of the Nabarro-Herring creep. For simplicity, this component is not considered. In summary, the proposed model accounts for DM creep including dislocation glide or glide-climb-glide and Nabarro-Herring creep for the inelastic behaviour of a SnAgCu crystal.

In terms of thermal expansion, the properties of a crystal can be described by a CTE tensor $\alpha$, which has 6 independent parameters. The relationship between the respective strain rate and temperature rate is expressed as

$$
\dot{\boldsymbol{\varepsilon}}_{\mathrm{th}}=\boldsymbol{\alpha} \dot{T}
$$

\subsection{Integration}

In the applied finite element software ABAQUS/Standard, a user subroutine, UMAT, is developed to implement the suggested material model. In this subroutine, two types of outputs are required:

(1) Update stresses $\boldsymbol{\sigma}$;

(2) Update material Jacobian matrix $\mathbf{D}$, where $\mathbf{D}=\frac{\partial \Delta \boldsymbol{\sigma}}{\partial \Delta \boldsymbol{\varepsilon}}$.

Details of this subroutine can be found in [8]. The following integration procedures are to provide these two types of outputs based on the method suggested in [9].

For the dislocation creep, the rate of shear strain $\dot{\gamma}^{(\alpha)}$ for a given slip system $\alpha$ is defined 
by:

$$
\dot{\gamma}^{(\alpha)}=\frac{d \gamma^{(\alpha)}}{d t} .
$$

Employing a linear interpolation, the increment of shearing strain within a time increment $\Delta t$ is defined by

$$
\left.\Delta \gamma^{(\alpha)}=\Delta t \mid(1-\theta) \dot{\gamma}_{t}^{(\alpha)}+\theta \dot{\gamma}_{t+\Delta t}^{(\alpha)}\right\rfloor,
$$

where $\theta$ ranges from 0 to 1 . Since the shear rate is a function of the shearing stress and temperature as shown in Eq. (7), its Taylor expansion gives

$$
\gamma_{t+\Delta t}^{(\alpha)}=\dot{\gamma}_{t}^{(\alpha)}+\frac{\partial \dot{\gamma}_{t}^{(\alpha)}}{\partial \tau^{(\alpha)}} \Delta \tau^{(\alpha)}+\frac{\partial \dot{\gamma}_{t}^{(\alpha)}}{\partial T} \Delta T
$$

where $\Delta T$ and $\Delta \tau^{(\alpha)}$ are the increments of temperature and shearing stress in slip system $\alpha$, respectively. Substituting Eq. (13) into Eq. (12), the increment of shearing strain is rearranged:

$$
\Delta \gamma^{(\alpha)}=\Delta t\left[\dot{\gamma}_{t}^{(\alpha)}+\theta \frac{\partial \dot{\gamma}_{t}^{(\alpha)}}{\partial \tau^{(\alpha)}} \Delta \tau^{(\alpha)}+\theta \frac{\partial \dot{\gamma}_{t}^{(\alpha)}}{\partial T} \Delta T\right] .
$$

Following the same procedure, the increment of the creep component due to vacancy diffusion is defined as

$$
\Delta \varepsilon_{\mathrm{vd}}^{i j}=\Delta t\left[\dot{\varepsilon}_{\mathrm{sd} t}^{i j}+\theta \sum_{k, l} \frac{\partial \dot{\varepsilon}_{\mathrm{vd} t}^{i j}}{\partial \sigma^{k l}} \Delta \sigma^{k l}+\theta \frac{\partial \dot{\varepsilon}_{\mathrm{vd} t}^{i j}}{\partial T} \Delta T\right] .
$$

The increment of the stress is determined by Eqs. (1), (2) and (3):

$$
\Delta \boldsymbol{\sigma}=\mathbf{C}:\left(\Delta \boldsymbol{\varepsilon}_{\text {total }}-\Delta \boldsymbol{\varepsilon}_{\mathrm{dc}}-\Delta \boldsymbol{\varepsilon}_{\mathrm{vd}}-\Delta \boldsymbol{\varepsilon}_{\mathrm{th}}\right)
$$

Combining Eqs. (5) and (16), we get: 


$$
\Delta \boldsymbol{\sigma}=\mathbf{C}:\left(\Delta \boldsymbol{\varepsilon}_{\text {total }}-\Delta \boldsymbol{\varepsilon}_{\mathrm{vd}}-\Delta \boldsymbol{\varepsilon}_{\mathrm{th}}-\sum_{\beta=1}^{N} \Delta \gamma^{(\beta)} \mathbf{s}^{(\beta)}\right)
$$

Substituting Eq. (17) into Eq. (8), we get:

$$
\Delta \tau^{(\alpha)}=\mathbf{s}^{(\alpha)}: \mathbf{C}:\left(\Delta \boldsymbol{\varepsilon}_{\text {total }}-\Delta \boldsymbol{\varepsilon}_{\mathrm{vd}}-\Delta \boldsymbol{\varepsilon}_{\mathrm{th}}-\sum_{\beta=1}^{N} \Delta \gamma^{(\beta)} \mathbf{s}^{(\beta)}\right)
$$

Substituting Eq. (18) into Eq. (14), we get:

$$
\begin{aligned}
& \theta \Delta t \frac{\partial \dot{\gamma}_{t}^{(\alpha)}}{\partial \tau^{(\alpha)}} s_{i j}^{(\alpha)} C_{i j k l} \Delta \varepsilon_{\mathrm{vd}}^{k l}+\sum_{\beta}\left(\delta_{\alpha \beta}+\theta \Delta t \frac{\partial \dot{\gamma}_{t}^{(\alpha)}}{\partial \tau^{(\alpha)}} s_{i j}^{(\alpha)} C_{i j k l} s_{k l}^{(\beta)}\right) \Delta \gamma_{\beta}= \\
& \Delta t \dot{\gamma}_{t}^{(\alpha)}+\theta \Delta t \frac{\partial \dot{\gamma}_{t}^{(\alpha)}}{\partial T} \Delta T+\theta \Delta t \frac{\partial \dot{\gamma}_{t}^{(\alpha)}}{\partial \tau^{(\alpha)}} s_{i j}^{(\alpha)} C_{i j k l}\left(\Delta \varepsilon_{\mathrm{th}}^{k l}-\Delta \varepsilon_{\mathrm{th}}^{k l}\right)
\end{aligned}
$$

where $\delta_{\alpha \beta}$ is the Kronecker delta. Substituting Eq. (17) into Eq. (15), we get:

$$
\begin{aligned}
& \Delta \varepsilon_{\mathrm{vd}}^{a b}+\theta \Delta t \frac{\partial \dot{\varepsilon}_{\mathrm{vd} t}^{a b}}{\partial \sigma^{i j}} C_{i j k l} \Delta \varepsilon_{\mathrm{vd}}^{k l}+\theta \Delta t \sum_{\alpha=1}^{N} \frac{\partial \dot{\varepsilon}_{\mathrm{vd} t}^{a b}}{\partial \sigma^{i j}} C_{i j k l} s_{k l}^{(\alpha)} \Delta \gamma_{\alpha}= \\
& \Delta t \dot{\varepsilon}_{\mathrm{vd} t}^{a b}+\theta \Delta t \frac{\partial \dot{\varepsilon}_{\mathrm{vd} t}^{a b}}{\partial T} \Delta T+\theta \Delta t \frac{\partial \dot{\varepsilon}_{\mathrm{vd} t}^{a b}}{\partial \sigma^{i j}} C_{i j k l}\left(\Delta \varepsilon_{\mathrm{total}}^{k l}-\Delta \varepsilon_{\mathrm{th}}^{k l}\right)
\end{aligned}
$$

According to Eqs. (19) and (20), if the total strain increment is known, the increment of each inelastic strain component can be determined. Substituting these strain increments into Eq. (17), the increment of stresses is obtained, which can be used to update stresses. Let us note that the material Jacobian matrix adopted in this paper is the elastic matrix. It should be mentioned that properties of SnAgCu crystal are not homogenous within a grain since the distribution of $\mathrm{Cu}_{6} \mathrm{Sn}_{5}$ and $\mathrm{Ag}_{3} \mathrm{Sn}$ intermetallic compounds (IMCs) is not uniform in $\beta$-Sn matrix. These IMCs can block the movement of dislocations during deformation, leading to the hardening effect. In a general isotropic constitutive equation, a threshold stress is defined to account for this effect [10]. However, in the model for a crystal, the threshold stress is different in each slip system since it is related to the Burgers vector. This will result in the 
number of unknown parameters equal to the number of slip systems. To simplify the model, the effect of these substructures within a grain is neglected.

\section{Finite element model}

\subsection{Model Geometry}

The model geometry for the flip chip package is based on components FC317G5.08C254DC and 903001 from Topline. Figure 2 shows the geometry of the flip chip. The dimensions of the silicon chip are $5.08 \mathrm{~mm} \times 5.08 \mathrm{~mm}$ with thickness $0.625 \mathrm{~mm}$. Solder bumps are distributed in an area array under the silicon chip with spacing $0.254 \mathrm{~mm}$. The height and diameter of a joint are $0.1 \mathrm{~mm}$ and $0.136 \mathrm{~mm}$, respectively. The substrate is made of the fourlayer FR4 printed circuit board with thickness $0.8 \mathrm{~mm}$. In order to reduce the influence of the edges of the board in calculations, its dimensions in plane in the model are relatively larger than that of the chip: $7.62 \mathrm{~mm} \times 7.62 \mathrm{~mm}$.

The FE mesh for the package is built in MSC.Marc/Mentat and transformed for ABAQUS with MSC.Patran. Due to symmetry of the package, one quarter of it is analyzed to enhance the calculation efficiency. Figure 3 shows the FE mesh of the model. A joint in the centre of the model is focused in this analysis and, therefore, has a finer mesh than other joints (Fig. 3b). The under-bumps metallization between the silicon chip and solder joints and metal finishes between solder joints and the substrate are neglected in the model, and three components are ideally bonded along an interface (each with diameter $0.11 \mathrm{~mm}$ ). On the symmetric sections of the flip chip package (middle sections along axis $\mathrm{X}$ and $\mathrm{Y}$ in Fig. 3), symmetric boundary conditions are applied.

\subsection{Material properties}

Since SnAgCu solder is more ductile than the silicon chip and FR4 substrate, the last two 
components are assumed to be in the elastic state during the entire loading process. In this case, three types of properties are required for these two materials: elastic modules, Poison's ratios and CTEs. Note that FR4 board has anisotropic properties. In order to have a a basis for comparison, a traditional creep model for a bulk specimen is also applied for SnAgCu solder joints. In this model, the elastic and thermal behaviour can be described in the same way as that for the crystal model, which is indicated in Eqs. (1), (3) and (10). For the creep component, its rate $\dot{\varepsilon}_{\mathrm{c}}$ is expressed in the form of a hyperbolic sin law [5]:

$$
\dot{\varepsilon}_{\mathrm{c}}=C_{1} \sinh \left(C_{2} \sigma_{\text {eq }}\right)^{n_{3}} \exp \left(-\frac{Q_{1}}{R T}\right),
$$

where $C_{1}$ and $C_{2}$ are the material constants, $n_{3}$ is the stress exponent, $Q_{1}$ is the activation energy $\left(C_{1}=7.9251 \times 10^{5}, C_{2}=3.56 \times 10^{-2}, n_{3}=6, Q_{1}=67.9 \mathrm{~kJ} / \mathrm{mole}, R=8.314 \times 10^{-3} \mathrm{~kJ} / \mathrm{mole}\right)$. In this paper, the dependence of elastic and thermal properties on temperature is not considered. The choice of magnitudes for material parameters is based on [11].

For the constitutive model for SnAgCu crystals, due to the lack of data for its parameters, its properties are derived from those of a bulk specimen. Since the discussion in this paper mainly focuses on the inelastic behaviour of a $\mathrm{SnAgCu}$ crystal, its elastic and thermal properties are assumed to be isotropic and the same as of a bulk specimen. The adopted parameters for VD creep are derived from the creep behaviour of a bulk specimens in the low stress region in [12] $\left(A=1.2 \times 10^{-1}, n_{2}=6.9, Q=106 \mathrm{~kJ} / \mathrm{mole}\right)$. As for dislocation creep, the stress exponent, activation energy and the constant $A^{(\alpha)}$ are assumed to be the same for all slip systems. These parameters are the same as those of a bulk solder's power law equation at the high stress region $\left(A^{(\alpha)}=1.52 \times 10^{-2}, n_{3}=11.6, Q^{(\alpha)}=93 \mathrm{~kJ} / \mathrm{mole}\right)$ [12].

\subsection{Loading history}


In a general numerical procedure to predict reliability of solder joints in electronics, a solder's response in a package under a thermal cyclic test is simulated by a FE model. Then the analysis results are used to predict the cycles to failure on the basis of a fatigue model [13]. This paper adopts the same type of load - cycling thermal loading - to demonstrate feasibility and the potential of the proposed model to predict a micro joint's behaviour in electronics. Since the package dimensions are rather small, the temperature gradient in it is negligible for the suggested loading type. The temperature is assumed to be homogenous in the package, and it changes with the ambient temperature. The heating and cooling rate of a thermal cycle is $10 \mathrm{~K} / \mathrm{min}$. Its maximum and minimum temperatures are $353 \mathrm{~K}$ and $253 \mathrm{~K}$, respectively. Figure 4 demonstrates the temperature change during a single cycle of the thermal cycling test.

\section{Results and discussion}

\subsection{Traditional model}

At the first stage of numerical simulations, the model for a bulk specimen (Eq. (21)) is applied to all solder joints to illustrate the deformational behaviour of the package during a thermal cycle and to make a reference for a further comparison. Our previous studies showed that the initial temperature has a little effect on these stabilized results [11]. Therefore, the initial cooling stage during manufacturing is neglected in this study, and the cycle starts at a room temperature (298 K). Figure 5 exhibits the distribution of the equivalent stress in the package when it reaches Point A (318 K, 120 s) during the first half cycle. The deformation of the package has been scaled by a factor 300. The figure clearly shows the warpage trend of the substrate when the temperature increases: its edges move upward along axis 3. This deformation is due to the difference in CTEs in the package plane (directions 1 and 2) between the silicon chip and substrate. Due to rigidity of the silicon chip (with an elastic modulus $131 \mathrm{GPa}$ ), when the temperature increases, the deformation is transferred through 
solder joints and absorbed by the soft substrate (also part of it is absorbed by solder joints). The constraint on expansion of the top surface of the substrate is considerably large, leading to an upward warpage of its edges along direction 3. The deformation trend of the silicon chip, which is not presented in the figure, is the same as that of the substrate, but its amplitude is much smaller. Between the substrate and silicon chip, solder joints experience a shearing deformation. Since the difference in displacements of the substrate and silicon chip at their edges is larger than at the centre, peripheral joints undergo a more serious deformation, resulting in larger residual stresses. For a single joint, stresses concentrate at solder/substrate and solder/chip interfaces as shown in Fig. 5. This is due to sharp angles of connections at these interfaces. Therefore, under a thermal cycling load, interfaces of a peripheral joint are one of the most critical positions in the package.

When the temperature decreases, the substrate has in an opposite trend of deformation to that of the temperature increase. Edges of the substrate move downwards along direction 3 (as shown in Fig. 6), presenting the state at the minimum temperature of the first cycle. The substrate contracts in direction 3 with the decrease in temperature. But the extent of deformation is considerably higher than that in directions 1 and 2 since the FR4 board has anisotropic thermal properties: the CTE in the direction normal to the plane is higher than that in the plane. The stress distribution in solder joints shown in Fig. 6 is similar to that in Fig. 5: peripheral joints have larger equivalent stresses, and stresses mainly concentrate at interfaces. But the stress magnitudes are considerably higher. This is due to a creep behaviour of solder joints, which is able to release more stresses at higher temperature.

Figure 7 presents evolution of the equivalent stress with equivalent creep strain at point $\mathrm{C}$ of Fig. 5. It shows that the stress and strain can reach a stable stage after four cycles. Following a general procedure, the failure of this joint can be predicted by using the magnitude of the strain change for a stable cycle, as shown in Fig. 7, in the fatigue model. 


\subsection{Crystal model}

\subsubsection{Effect of thermal expansion}

The proposed crystal model for SnAgCu is firstly applied to the central joint (with a finer mesh as shown in Fig. 3) of the package. All the elements in this joint have the same lattice orientation, and therefore, it is considered as a single crystal. The orientation of the grain is arbitrarily chosen and its relation with the entire model is presented in Fig. 8. Other joints are still described by the traditional hyperbolic sin law as given in Eq. (4). In the crystal model, all the strain components, including elastic, creep and thermal expansion ones, are accounted. Here 16 activated slip systems (the same as in [4]) are considered for DM creep. Figure 9(a) presents the distribution of the equivalent stress in the single-crystal joint when the temperature increases to $318 \mathrm{~K}$ (Point A in Fig. 4). It shows that interfaces of the joint have the largest residual stresses. But there are two areas of stress concentration at the interface: in the front (Point D) and back areas (Point E) along the direction of shearing deformation (a bisector between directions 1 and 2), respectively. To study this distribution, all stress components are analyzed. It is found that the largest component is the normal stress $\sigma_{33}$, the level of which is an order of magnitude higher than that of other components. Figure 9(b) gives the distribution of $\sigma_{33}$. It shows that the absolute maximum value of $\sigma_{33}$ is close to that of the equivalent stress in Fig. 9(a), and that this component also peaks at Points D and E. According to the definition of von Mises equivalent stress, $\sigma_{33}$ is the major source of stress concentration in Fig. 9(a). Refocusing on $\sigma_{33}$, it is found that its value is positive at Points D and negative at E, indicating that these two points experience tension and compression during the shearing deformation with the increase in temperature. Since the absolute value of $\sigma_{33}$ at Points D and E is similar, the total normal load in direction 3 on interfaces is close to zero. In order to investigate the contribution of thermal expansion, the thermal component $\varepsilon_{\text {th }}$ in Eq. (1) 
is deactivated for the single-crystal joint. Other joints, as well as silicon chip and the substrate retain this component. The distribution of the equivalent stress when the temperature increases to Point A (318 K) is given in Fig. 9(c). Compared with Fig. 9(b), there is only one zone of stress concentration (Point F) at the interface, and its magnitude is higher. These changes result from the lack of the thermal component in this description of the single-crystal joint. With the temperature increase, the distance between the substrate and silicon chip increases due to the expansion of large number of peripheral joints in direction 3 . This opening leads to an additional tensile load acting on the single-crystal joint. Therefore, the tensile stress at point $\mathrm{B}$ increases with the compressive stress at point $\mathrm{C}$ reducing in direction 3, as shown in Fig. 9(d). Since this normal stress $\sigma_{33}$ is the major source of the equivalent stress, residual stresses increase at point $\mathrm{D}$ while reduce at point $\mathrm{E}$, leading to only one stress concentration zone at the interface. Another change is that the single crystal joint becomes thinner in directions 1 and 2 as shown in Fig. 9. Obviously, this results from the lack of expansion in these two directions. The additional elongation of the joint in direction 3 also contributes to this change since it leads to the contraction in directions 1 and 2 to maintain the volume of the joint.

\subsubsection{Effect of Vacancy Diffusion}

To analyze the influence of VD and make a comparison, all the strain components in Eqs. (1) and (2) are accounted at this stage of numerical simulation except VD creep $\varepsilon_{\mathrm{vd}}$,. Figure 10(a) presents the distribution of equivalent stress when the temperature increases to Point A in Fig. 4 (318 K). Compared with Fig. 9(a), which considers all strain components, the distribution is the same, including the value of the stress concentration. To investigate this 
phenomenon, the equivalent strain $\varepsilon_{\text {eq }}\left(\varepsilon_{\text {eq }}=\sqrt{\frac{2}{3} \boldsymbol{\varepsilon}: \boldsymbol{\varepsilon}}\right)$ due to DM and VD creep for Fig. 9(a) is given in Figs. 11(a) and (b), respectively. It can be seen that the deformation due to VD is considerably smaller, and therefore, has little effect. This is reasonable since the residual stress is relatively high for low temperatures. DM creep is the dominant deformation mechanism; when temperature increases, the deformation ability of DM creep is enhanced. As shown in Fig. 10 (b), the maximum stress is relaxed to $26.88 \mathrm{MPa}$ when the temperature reaches its maximum value, (373 K, Point B in Fig. 4). But this value is slightly higher than that in Fig. 12, which considers all strain components, indicating that VD does make a contribution to this relaxation. DM and VD creep accumulation at the maximum temperature (Point B in Fig. 4) is presented in Figs. 11 (c) and (d). It can be seen that compared with Figs. 11 (a) and (b) both of these two strains increase significantly, but the increase of VD creep is relatively larger (the VD creep increases by three orders of magnitude while DM creep increases by two orders). In the suggested model, VD is more sensitive to temperature due to its higher activated energy than DM (106 kJ/mole and $93 \mathrm{~kJ} /$ mole, respectively). Note that this comparison is a relative one. In this case, VD is more pronounced at high temperatures, leading to larger deformations. It is also found that the distribution of deformation due to these two creep mechanics is not the same as in Figs. 11(c) and (d). This is caused by their different deformation nature. DM creep can only be realised in certain slip systems. The largest deformation accumulates at a position where gliding is most convenient for some slip systems; in case of VD, it is directed by stresses. The highest deformation occurs in the area with highest stresses. Therefore, the distribution of deformation in Fig. 11 (d) is similar to that for the equivalent stress in Fig. 12.

In contrast to Chen's model [12], on which parameters the proposed model is based on, in Wiese's double power law for SnAgCu eutectic solder, the activation energy in a low-stress 
region is much lower than that in a high-stress region $(34.693 \mathrm{~kJ} / \mathrm{mole}$ and $61.193 \mathrm{~kJ} / \mathrm{mole}$, respectively) [7]. Assuming that Wiese's creep model at low stress (i.e. $A=1 \times 10^{-4}, n_{2}=3$ and $Q=34.6 \mathrm{~kJ} / \mathrm{mole}$ in Eq. (9)) is dominated by vacancy diffusion, it is directly applied to the studied case for comparison. To be consistent, the model parameters for DM creep and thermal expansion remain the same. Fig. 13(a) presents the equivalent stress distribution for the model with new parameters when temperature increases to Point A in Fig. 4. The stress distribution is similar to that in Fig. 9 (a), but the stress levels are lower. As can be seen, the maximum stress reduces from 29.11 MPa to 27.95 MPa, indicating that VD creep develops faster in Wiese's model than in Chen's one releasing more stresses. This is demonstrated by Fig. 13(d), which shows a considerably higher level of VD creep accumulation than that in Fig. 11(b). However, the accumulation of dislocation creep becomes less as shown in Figs. 13(c) and 11(a). These changes illustrate the interaction between DM creep and VD in the proposed model. As presented in Eq. (16), both types of creep can release stresses, which are the original driving force for both types of creep. If one leads to higher deformations, the reduced stresses will decrease the driving force of the other due to Eq. (7) or Eq. (9). To the contrary, the reducing deformation ability of one type of creep can lead to higher deformations due to the other. In the real case of deformation, DM and VD creep can also interact directly. For instance, a certain movement of vacancies is able to release dislocations, resulting in a softening effect. These reactions are not introduced into the model to avoid introduction of too many unknown parameters at this stage. When the temperature increases to the maximum value, Point B in Fig. 4, the accumulated deformation due to both DM and VD creep increases considerably as shown in Figs. 13(e) and 13(f). This increase for VD is more pronounced since it is more sensitive to temperature. Fig. 13(b) shows that the stresses are even lower than those in Fig. 12. This is due to the increasing deformation ability of VD in the case of Wiese's creep data. 
Similar to the traditional model, the single-crystal joint is tested for four thermal cycles. Parameters based on both Chen's and Wiese's creep models are used in computational studies. The relationship between the equivalent stress and equivalent total creep strain is presented in Fig. 14. It can be seen that both models can reach a stable state after four cycles. This demonstrates the ability of the proposed model to predict a response of micro-joints under a long-term thermal cycling in electronics.

\section{Conclusions}

In this paper, a constitutive model, which accounts for major mechanisms acting at high temperature in a crystal, is proposed for $\mathrm{SnAgCu}$ solder micro joints in electronics. It is implemented for a joint in a flip chip package. The obtained results show that:

1. Under a condition of varying temperature, thermal expansion is a major deformation mechanism for a single-crystal joint. It has a considerable effect on the stress state in the crystal, and also influences other mechanisms of deformation. Therefore, it cannot be neglected in estimates of the response of microelectronics package to thermal cycling.

2. To model the inelastic behaviour of solder micro joints, both dislocation creep and diffusional creep are introduced for a SnAgCu crystal. The implemented model shows that the development of one of the mechanisms can enhance the total creep. However, it reduces the effect of the other type of creep. The relevant sensitivity of deformation to temperature of these two types of creep is determined by their activation energy: higher activation energy indicates higher sensitivity.

3. The proposed model employing both Wiese's and Chen's creep datum is used to simulate four thermal cycles. The transition to a stable state during this loading for both formulations demonstrates the convergence ability of the proposed model. 


\section{Acknowledgements}

Financial support by the Engineering and Physical Sciences Research Council's Innovative Manufacturing and Construction Research Centre at Loughborough University under GR/R64483/01P is gratefully acknowledged. The financial support by the Alexander Von Humboldt Foundation is also gratefully appreciated.

\section{Reference}

[1] J. Gong, C. Liu, P.P. Conway, V.V. Silberschmidt, Crystallographic structure and mechanical behaviour of $\mathrm{SnAgCu}$ solder interconnects under a constant loading rate In: Electronics Components and Technology Conference (ECTC'57), John Ascuaga’s Nugget, Reno, Nevada (2007) in press.

[2] J. Gong, C. Liu, P.P. Conway, V.V. Silberschmidt, Grain features of SnAgCu solder and their effect on mechanical behaviour of micro-joints, In: Electronics Components and Technology Conference (ECTC'56), San Diego (2006) 250-257.

[3] M.A. Matin, E.W.C. Coenen, W.P. Vellinga, M.G.D. Geers, Correlation between thermal fatigue and thermal anisotropy in a Pb-free solder alloy, Scripta Mater. 53 (2005) 927-932.

[4] J. Gong, C. Liu, P.P. Conway, V.V. Silberschmidt, Micromechanical modelling of SnAgCu solder joint under cyclic loading: effect of grain orientation, Comput. Mater. Sci. 39 (2007) 187-197.

[5] V. Berdichevsky , P. Hazzledine, B. Shoykhet, Micromechanics of diffusional creep, Int. J. Eng. Sci. 35 (1997) 1003-1032.

[6] J.P. Clech, Review and analysis of lead-free solder material properties. Available from: <http://www.metallurgy.nist.gov/solder/>. 
[7] S. Wiese, K.-J. Wolter, Microstructure and creep behaviour of eutectic SnAg and SnAgCu solders, Microelectron. Reliab. 44 (2004) 1923-1931.

[8] Hibbitt, Karlsson, Sorensen, ABAQUS Analysis User’s Manual. Version 6.2, Pawtucket, Rhode Island, 2001.

[9] Y. Huang, A user-material subroutine incorporating single crystal plasticity in the ABAQUS finite element program, Mech Report 178, Harvard University, 1991.

[10] J. Gong, C. Liu, P.P. Conway, V.V. Silberschmidt, Modelling of Ag3Sn coarsening and its effect on creep of Sn-Ag eutectics, Mater. Sci. Eng. A 427 (2006) 60-68.

[11] J. Gong, C. Liu, P.P. Conway, V.V. Silberschmidt, Analysis of stress distribution in SnAgCu solder joint, Appl. Mech. Mater. 5-6 (2006) 359-366.

[12] Z. Chen, Y. Shi, Z. Xia, Constitutive relations on creep for SnAgCuRE lead-free solder joints, J. Electron. Mater. 33 (2004) 964-971

[13] W.W. Lee, L.T. Nguyen, G.S. Selvaduray, Solder joint fatigue models: review and applicability to chip scale packages, Microelectron. Reliab. 40 (2000) 231-244. 


\section{Figure Captions}

Fig. 1 Relationships between creep strain rate and stress for SnAgCu solder

Fig. 2 Geometry of flip chip package: a) entire package; b) package without silicon chip.

Fig. 3 FE mesh for flip chip package: a) quarter of entire package; b) mesh without silicon chip.

Fig. 4 Single cycle of thermal cycling test. Point A: $318^{\circ}$ K, 120 ss; Point B: 373 K, 450 s.

Fig. 5 Equivalent residual stresses in package (with removed silicon chip) after temperature increases to $318^{\circ} \mathrm{K}$ in the first cycle. Scale factor for deformation is 300 .

Fig. 6 Equivalent residual stresses in package (with removed silicon chip) after cooling down to $273^{\circ} \mathrm{K}$ in the first cycle. Scale factor for deformation is 50 .

Fig. 7 Relationship between equivalent stress and equivalent creep strain at Point $\mathrm{C}$ in Fig. 5 in first 4 cycles

Fig. 8 Relationship between orientation of single crystal joint's Sn matrix and coordinates for FE model of flip chip package

Fig. 9 Stress distribution in single crystal joint at the centre of the package (with finer mesh in Fig. 3), for $318^{\circ} \mathrm{K}$ (Point $\mathrm{A}$ in Fig. 4): (a) equivalent stress with thermal expansion; (b) stress component $\sigma_{33}$ with thermal expansion; (c) equivalent stress without thermal expansion; (d) stress component $\sigma_{33}$ without thermal expansion. Scale factor for deformation is 300 .

Fig. 10 Distribution of equivalent stress for a case without vacancy diffusion component in Eq. (2). (a) at Points A and (b) at point B in Fig. 4. Scales factors for deformation: (a) 300 and (b) 20.

Fig. 11 Distribution of equivalent strain (all strain components are considered): (a) dislocation creep, $318^{\circ} \mathrm{K}$; (b) vacancy diffusion, $318 \mathrm{~K}$; (c) dislocation creep, $373 \mathrm{~K}$; 
(d) vacancy diffusion, $373 \mathrm{~K}$. The deformation scale factor is 300 for (a) and (b), and 20 for (c) and (d)

Fig. 12 Equivalent stress distribution for Point B in Fig. 4. All strain components are considered. Scale factor for deformation is 20 .

Fig. 13 Equivalent stress and strain distribution based on Wiese's model: (a) stress, $318 \mathrm{~K}$; (b) stress, 373 K; (c) DM, 318 K; (d) VD, 318 K; (e) DM, 373 K; (f) VD, 373 K. The deformation scale factor is 300 for (a), (c) and (d), and 20 for (b), (e) and (f).

Fig. 14 Relationship between equivalent stress and total creep strain in the first 4 cycles at Point C in Fig. 5. Parameters for crystal model are based on Wiese’s (a) and Chen’s (b) models. 


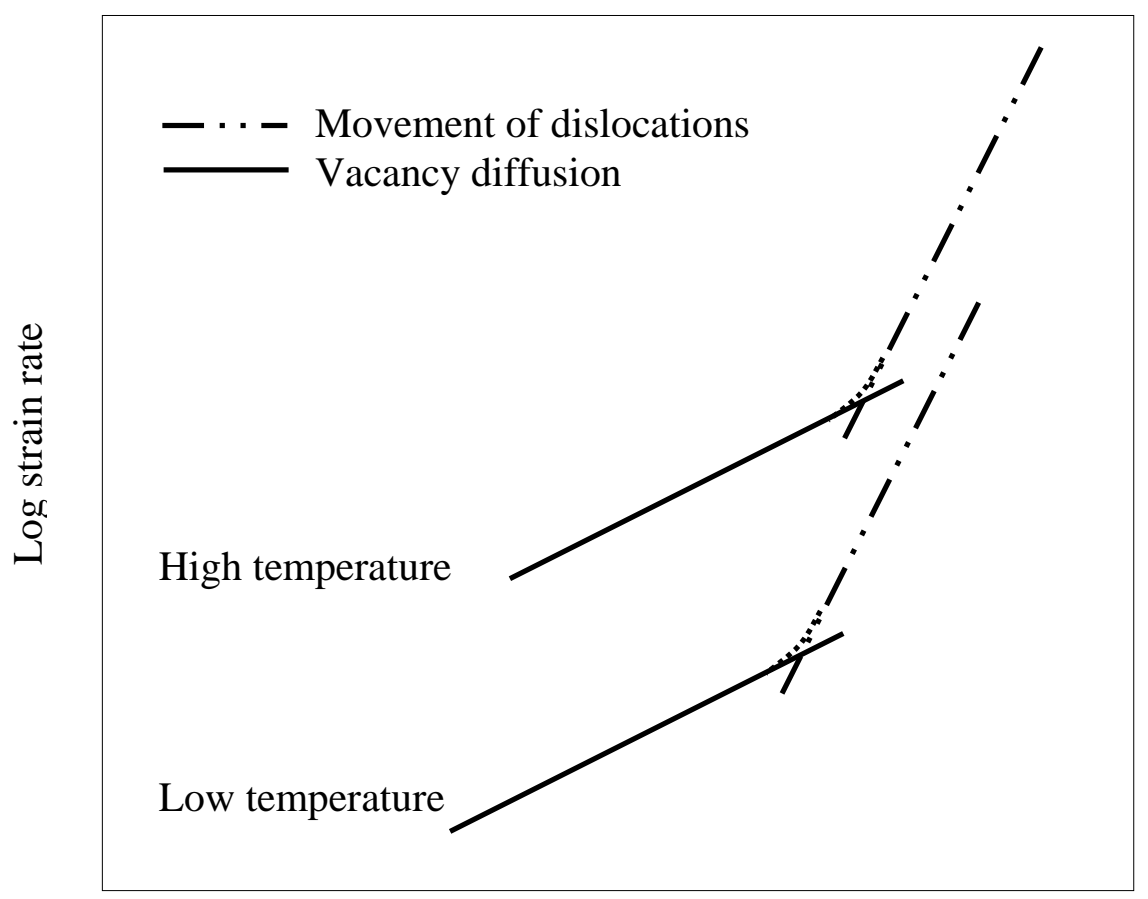

Log stress

Figure 1 
a)

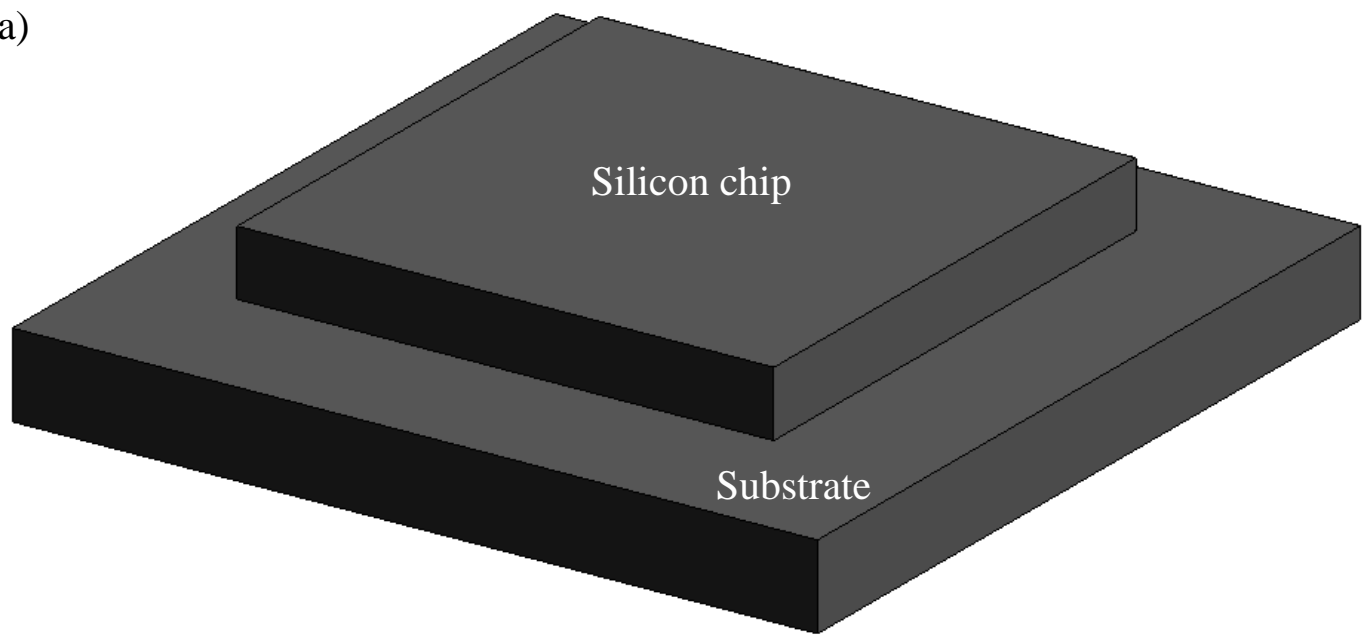

${ }^{3} r^{2} 1$

b)

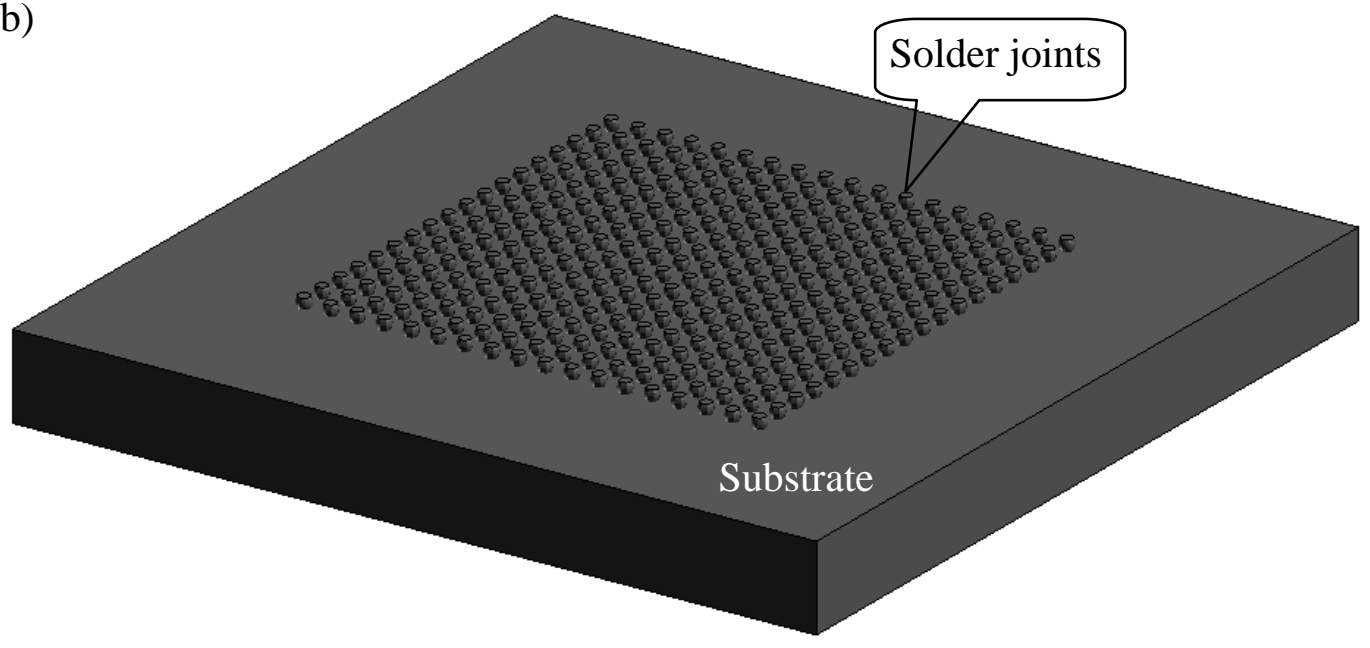

${ }^{3} r^{2} 1$

Figure 2 

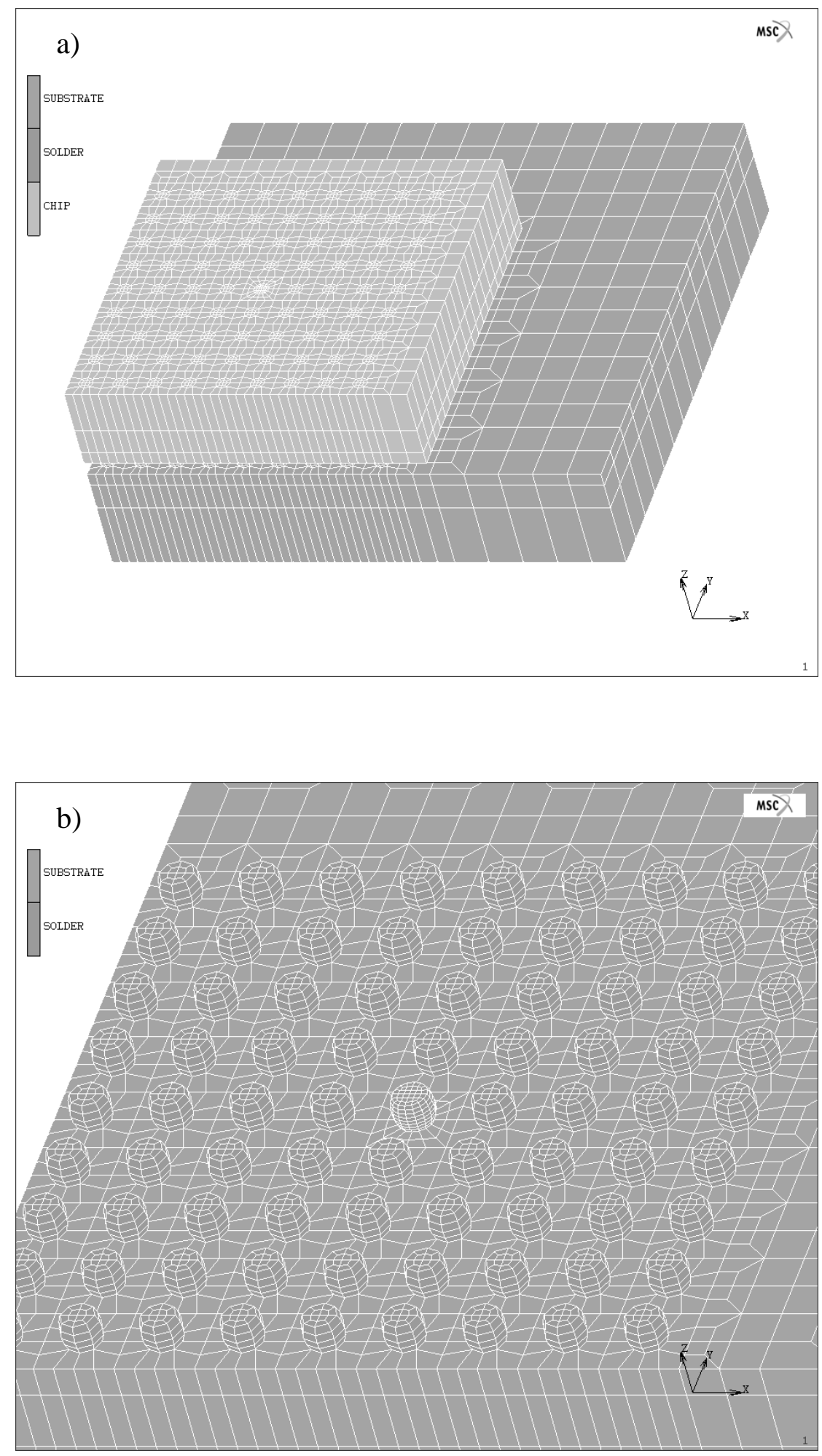

Figure3 


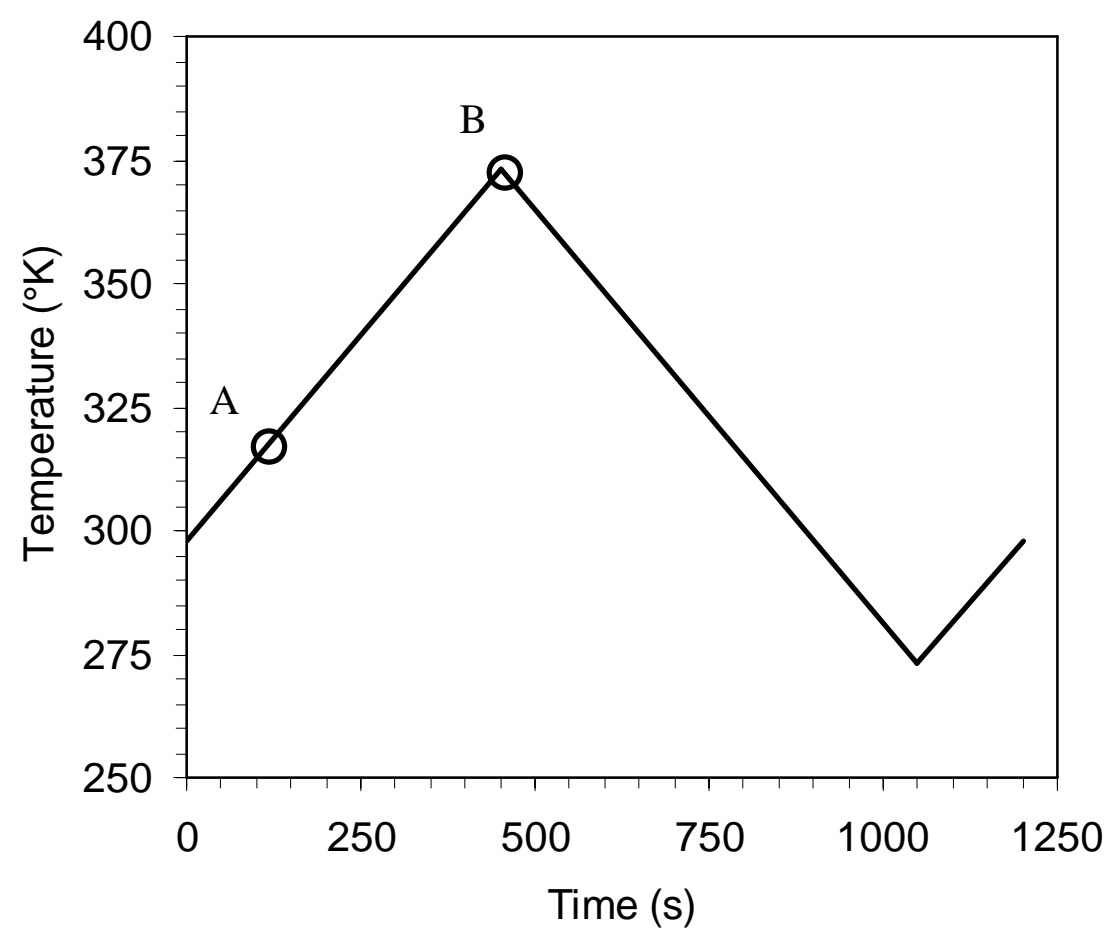

Figure 4 


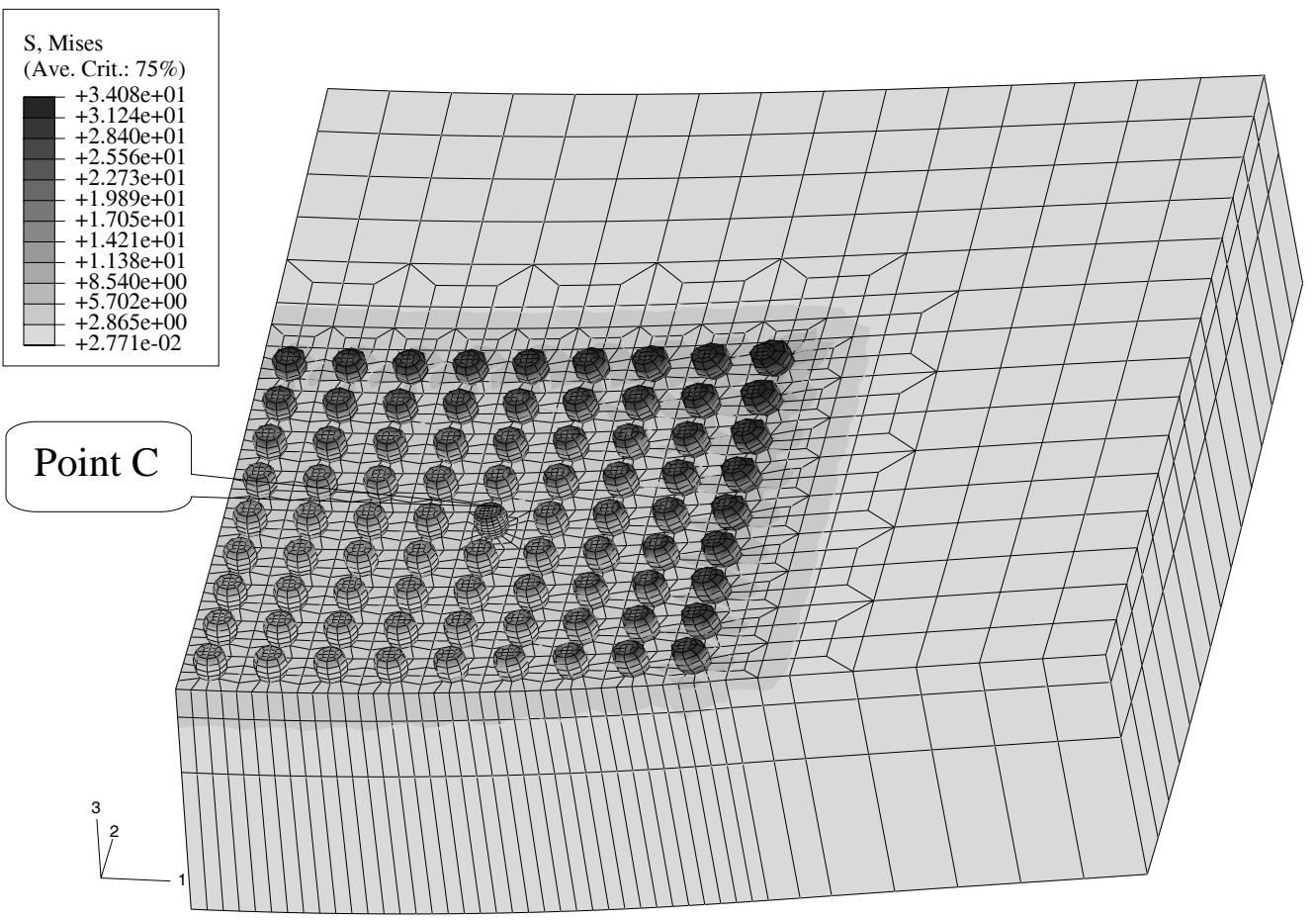

Figure 5 


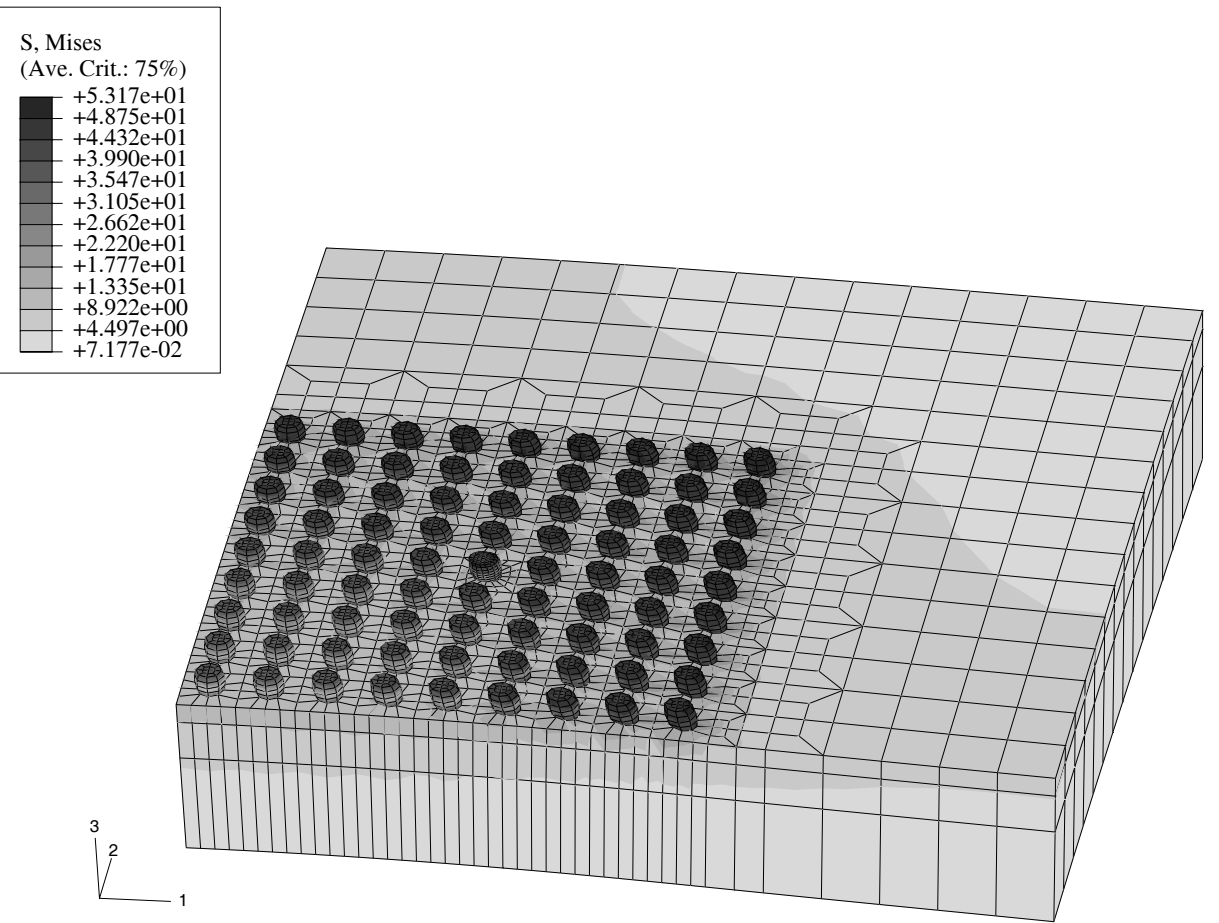

Figure 6 


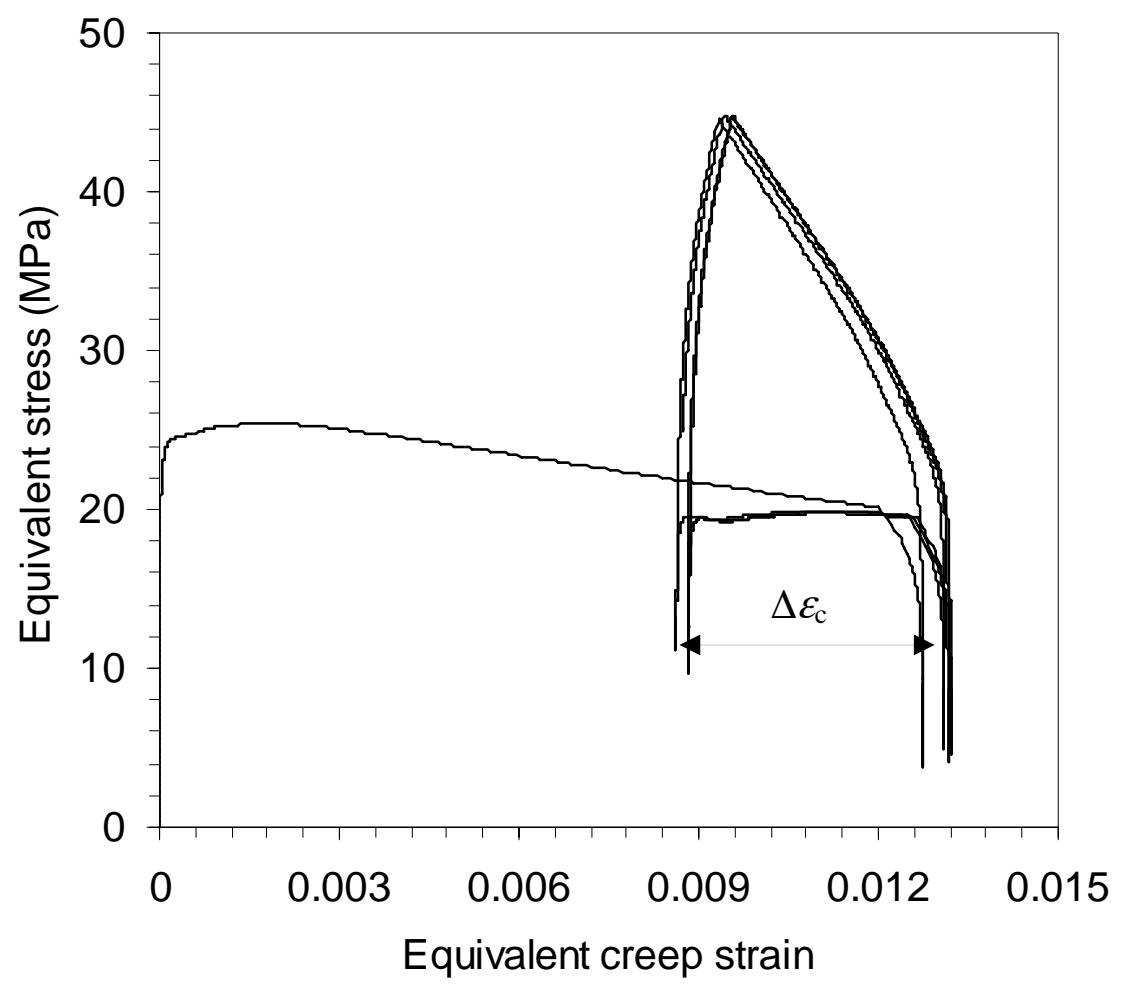

Figure 7 


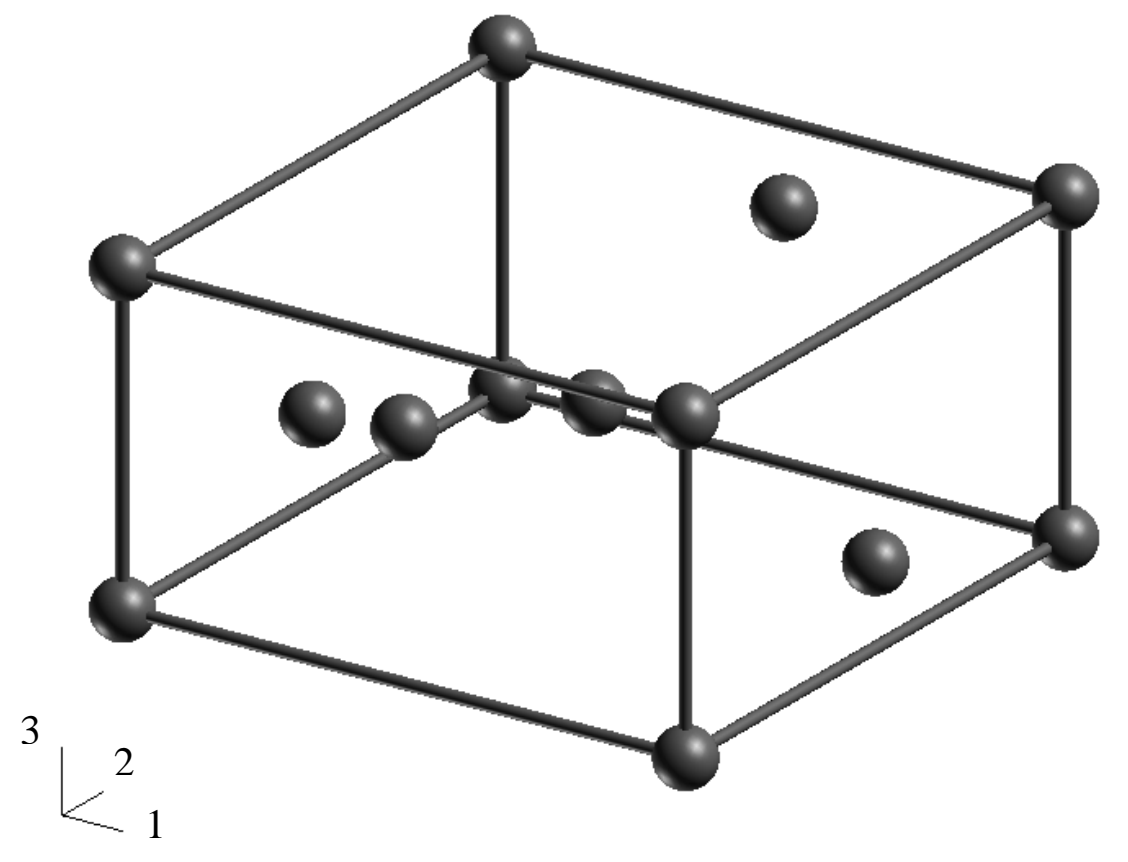

Figure 8 
(a)

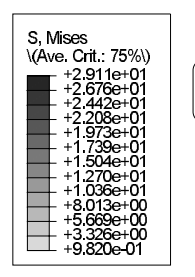

(c)
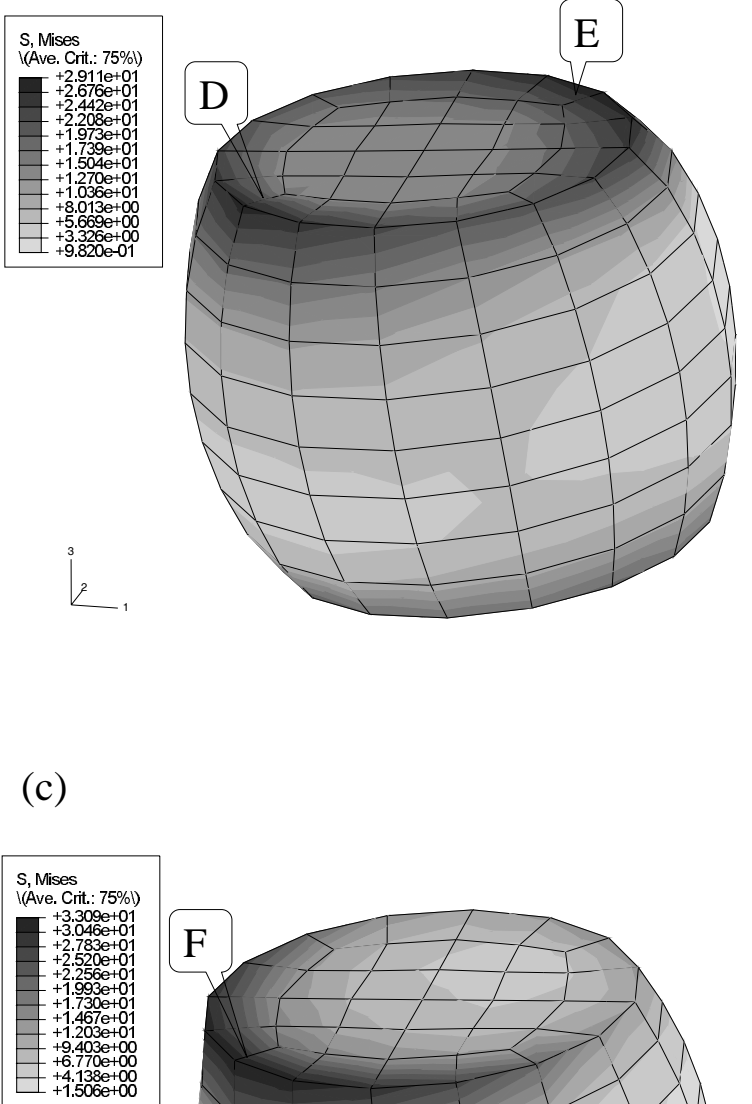

$\sum^{3}$ (b)
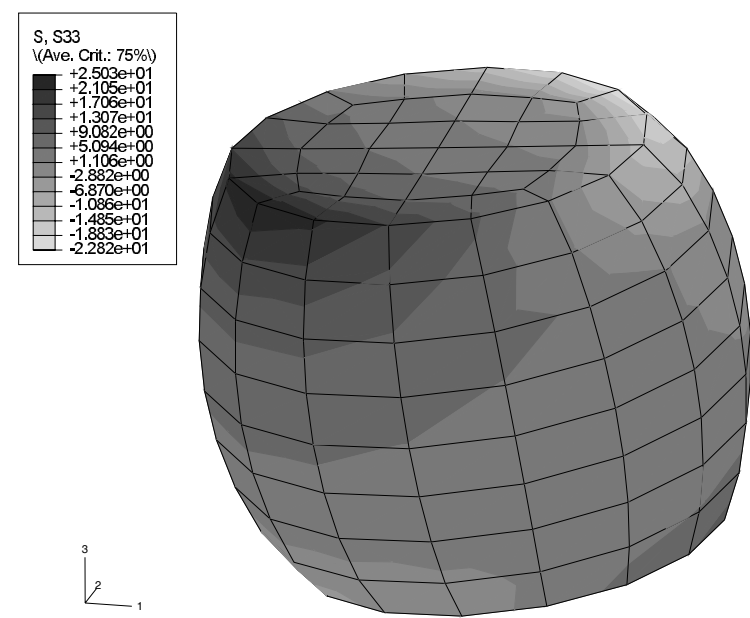

(d)
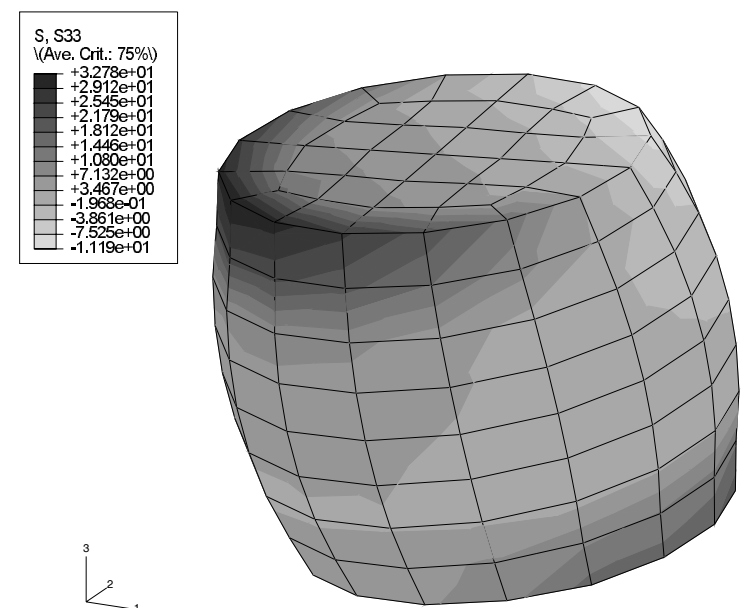

Figure 9 


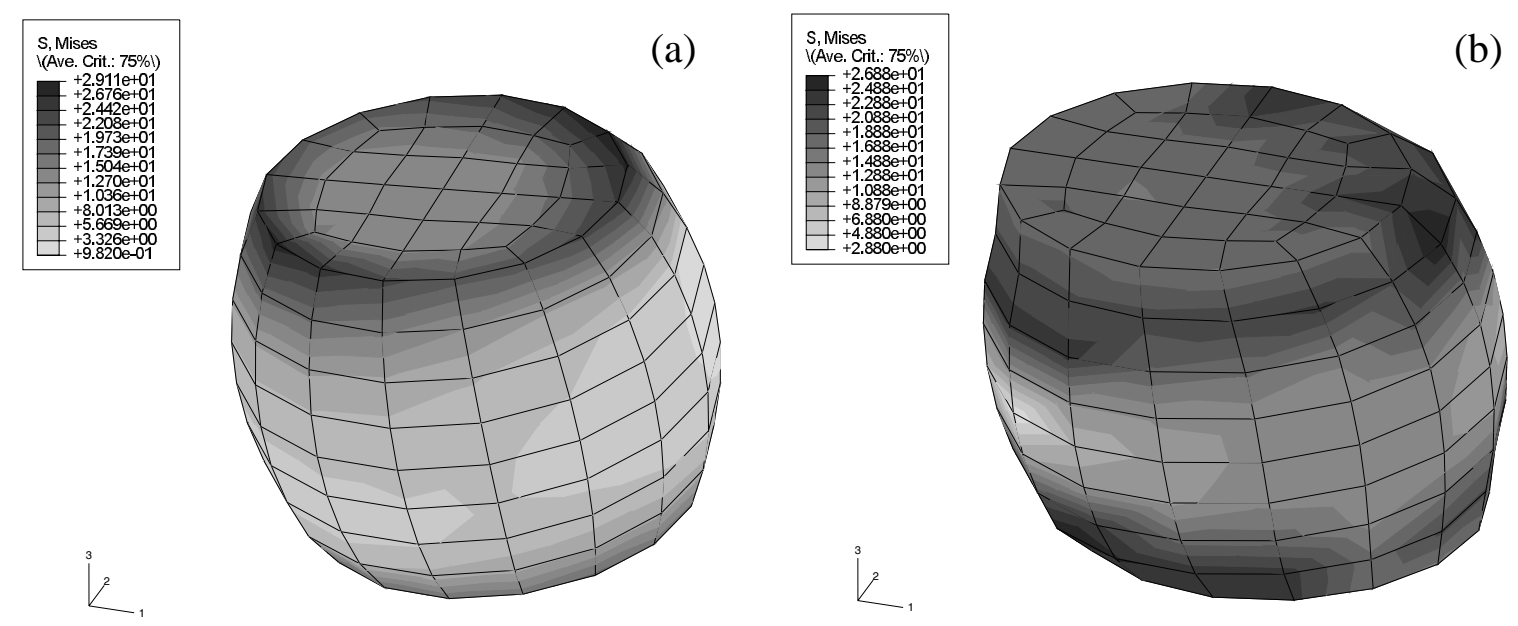

Figure 10 
(a)

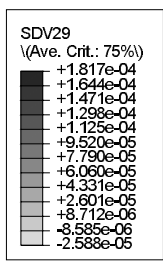

$2^{3}$

(c)

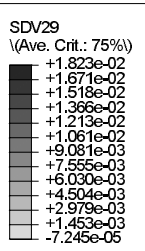

$i^{3}$
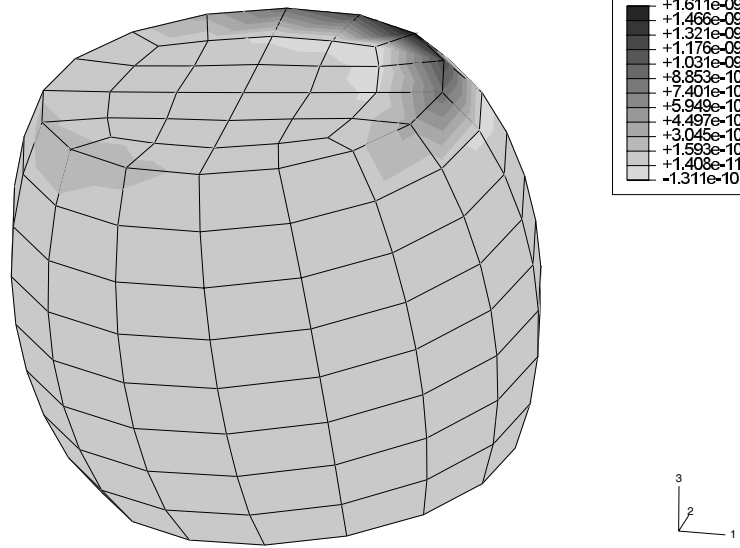

(d)
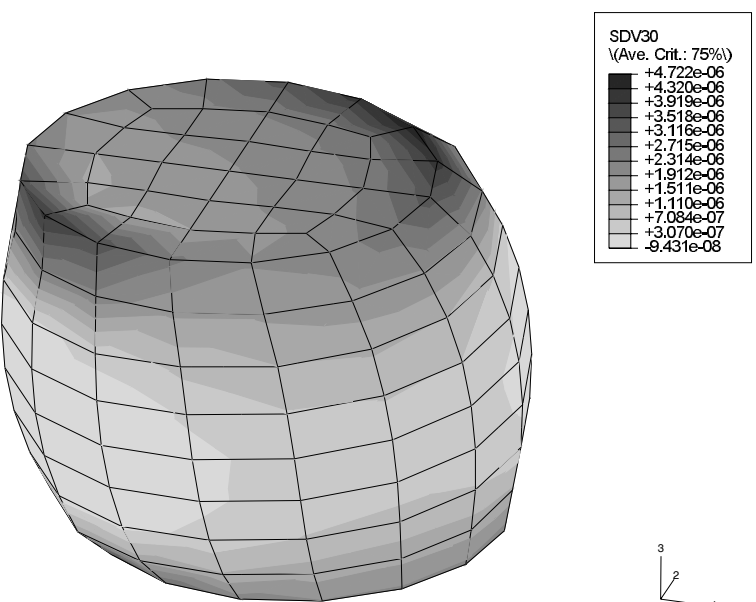

(b)
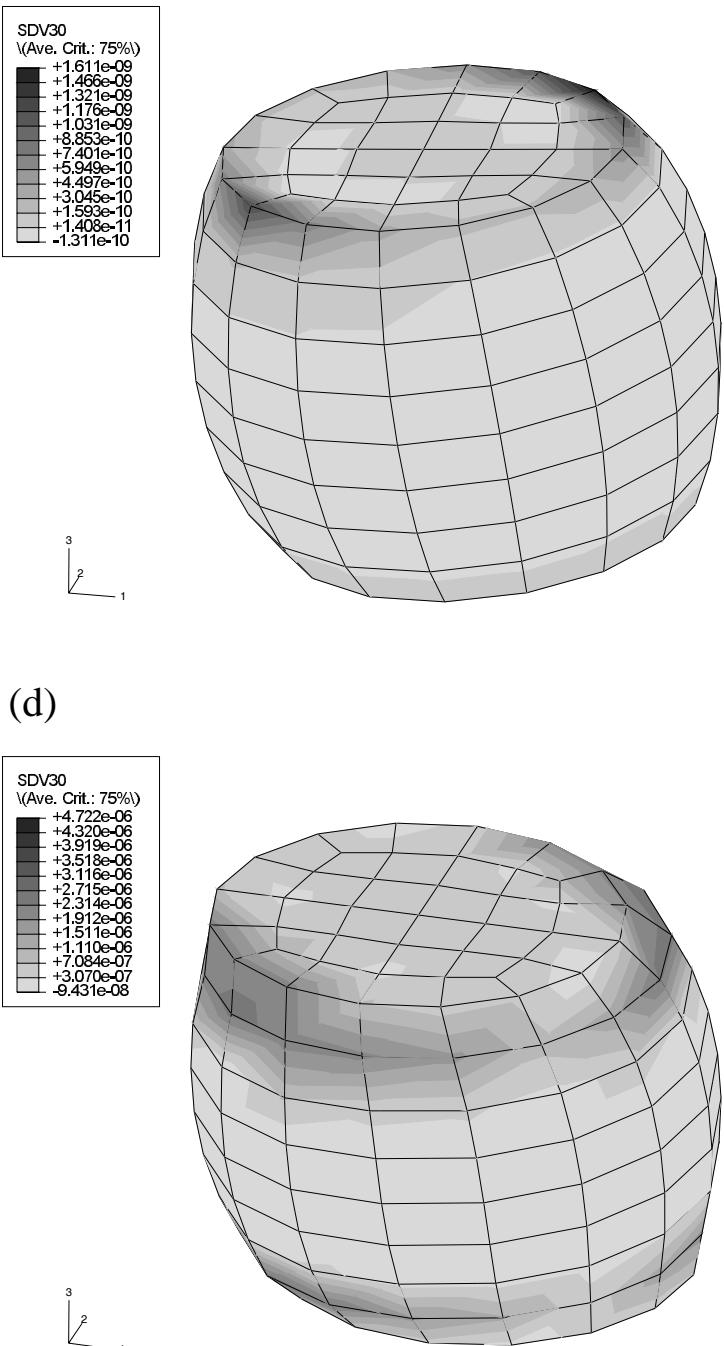

Figure 11 


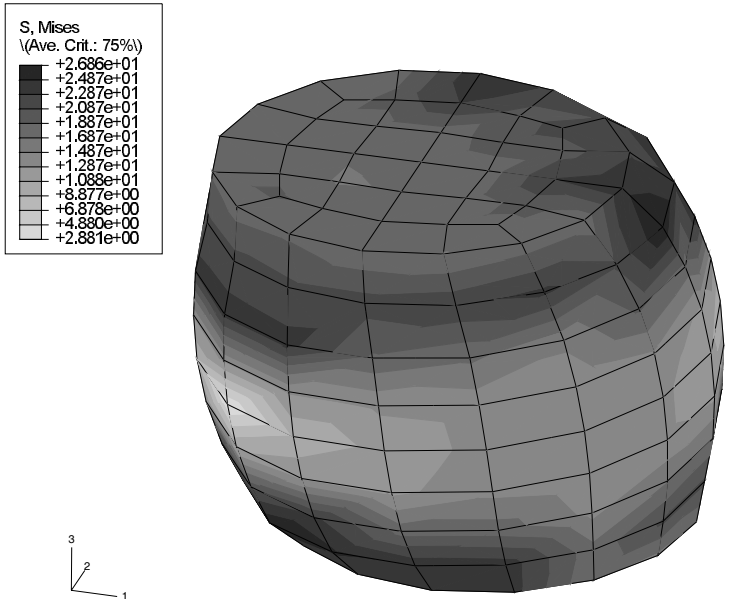

Figure 12 
(a)
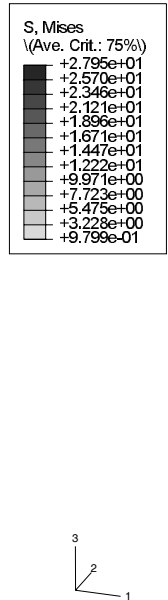

(c)
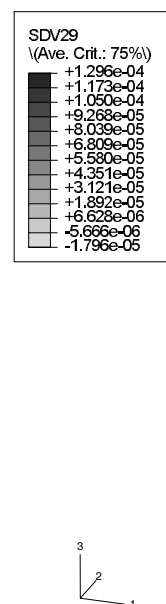

(e)

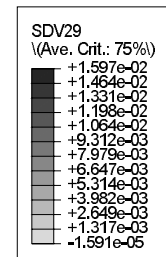

$2^{3}$

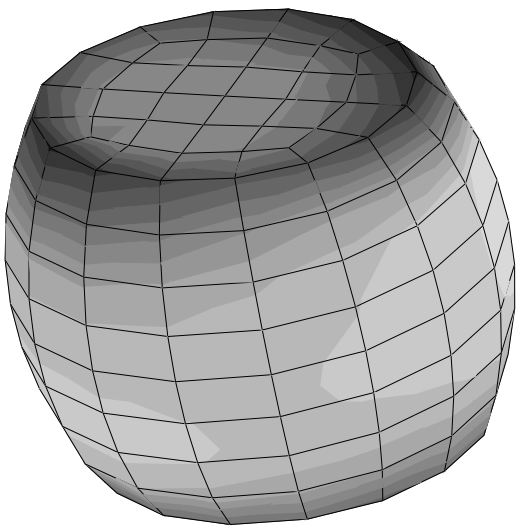

(d)
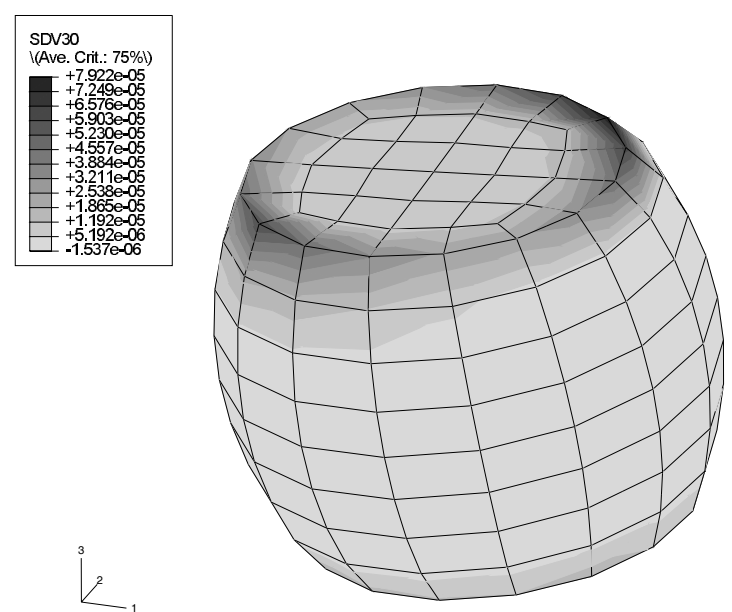

(f)
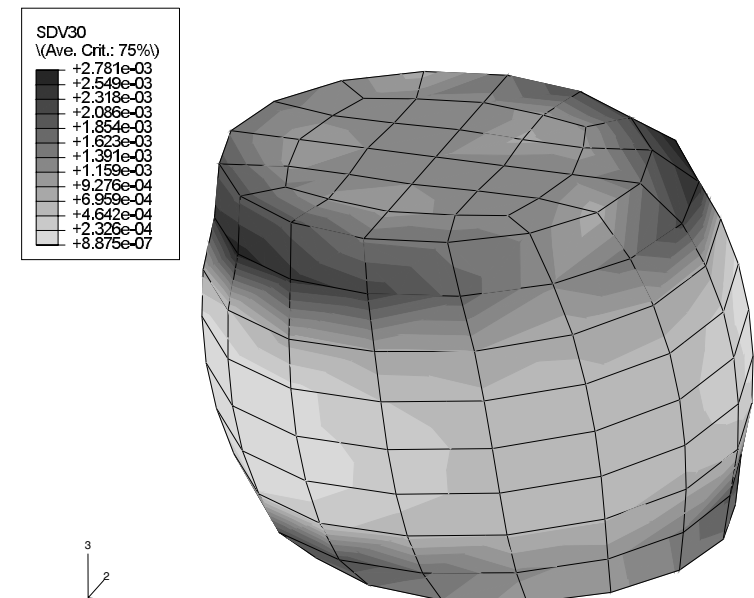

Figure 13 
(a)

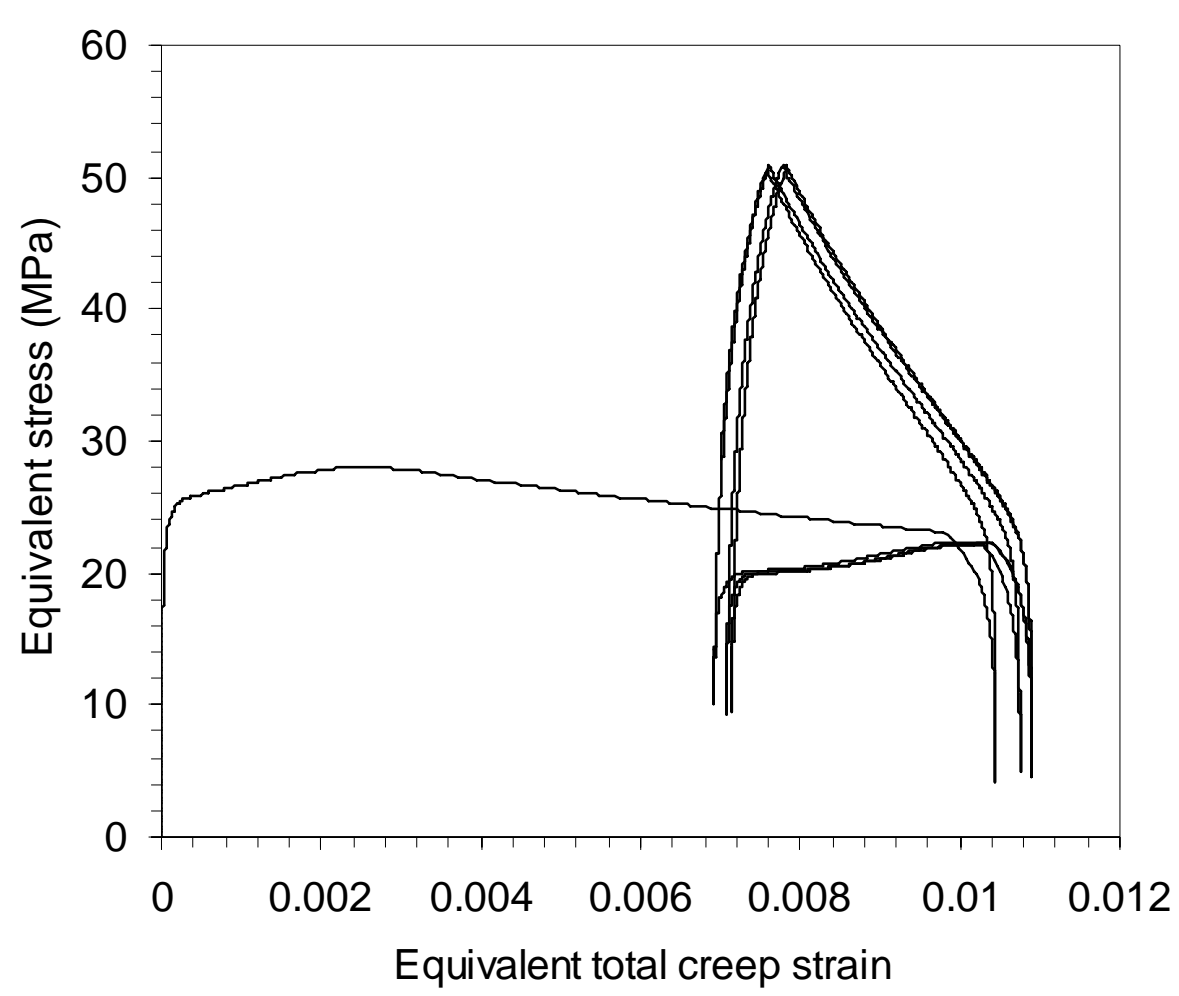

(b)

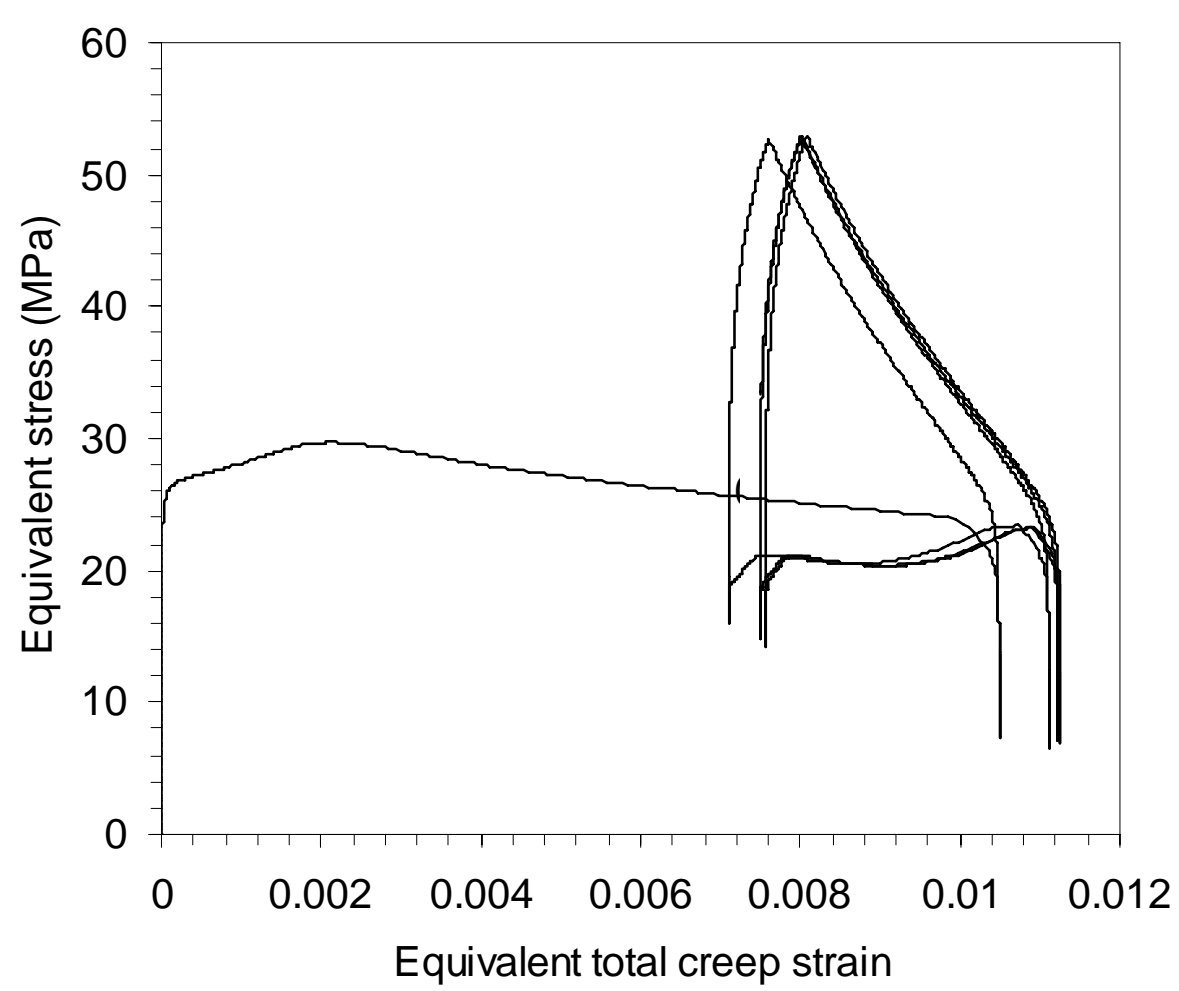

Figure 14 